\title{
Managing Vesicoureteral Reflux in the Pediatric Patient: a Spectrum of Treatment Options for a Spectrum of Disease
}

Tanya D. Davis, $M D^{*}$
H. Gil Rushton, $M D$

\section{Address}

*Division of Pediatric Urology, Children's National Medical Center, 111 Michigan Ave NW, Washington, DC, 20010, USA

Email: tandavis@childrensnational.org

Published online: 9 February 2016

(C) Springer International Publishing AG 2016

This article is part of the Topical Collection on Pediatric Urology

Keywords Vesicoureteral reflux $\cdot$ Urinary tract infection - Pediatrics $\cdot$ Antibiotic prophylaxis $\cdot$ Deflux $\cdot$ Ureteral reimplantation $\cdot$ Bowel and bladder dysfunction

\section{Opinion statement}

Vesicoureteral reflux (VUR), or the reverse flow of urine from the bladder into the ureter or renal collecting system, is characterized by a wide spectrum of severity. Consequently, a spectrum of treatment options exists that can be broadly divided into non-operative and surgical management. Non-operative management is based on the natural history of reflux which suggests that the vast majority of VUR, and in particular low grades of reflux, will resolve spontaneously. Furthermore, most patients with lower grades of VUR are at relatively low risk for recurrent pyelonephritis. The focus of non-operative management is to prevent urinary tract infections that, when combined with VUR, place a child's kidney at risk for renal damage and potential loss of renal function. This is typically achieved by optimizing bladder and bowel function through a combination of dietary, behavioral, or pharmacologic therapies and in some cases may include the use of antibiotic prophylaxis. Surgical management seeks to mechanically correct VUR, either by endoscopic injection of the intravesical ureteral tunnel with bulking agents (Deflux) or with open or minimally invasive surgical ureteral reimplantation. Deflux provides a less invasive but comparatively less successful alternative to surgical reimplantation, and therefore, surgical reimplantation is more frequently utilized in children with persistent high-grade VUR, known renal damage, and associated bladder and bowel dysfunction. These approaches are not mutually exclusive, and the management of VUR is highly individualized, taking into consideration a 
litany of factors including a child's age, sex, severity of reflux, response to previous therapy options, the presence and severity of renal damage, and concomitant bladder and bowel dysfunction. These considerations must also be balanced with patient/parent preference and the potential consequences of choosing a particular treatment strategy.

\section{Introduction}

Primary vesicoureteral reflux (VUR) describes reverse flow of urine from the urinary bladder into the ureter or renal collecting system as a result of abnormal development of the ureterovesical junction (UVJ). Although the exact incidence of primary or congenital VUR is difficult to determine due to its often asymptomatic nature, it is estimated to occur in approximately 1-9\% of all children and infants, $10-20 \%$ of infants with antenatal hydronephrosis, and $30-50 \%$ of children presenting with a UTI $[1,2,3 \bullet]$. While more commonly found in infant boys, as children age, VUR is more commonly detected in girls, probably because girls are at higher risk for UTI after the first 612 months of life. A familial component to VUR also exists, evidenced by the finding that approximately one third of siblings of a child with VUR will also have VUR [4]. VUR may also be secondary, i.e., acquired as sequelae of functional (e.g., neuropathic bladder, dysfunctional voiding) or anatomic (e.g., posterior urethral valves) bladder outlet obstruction. In secondary VUR, high intravesical pressures overcome the mechanism of the UVJ to prevent reverse flow of urine into the upper tracts, leading to the development of VUR. Regardless of the etiology, VUR in combination with UTI is a well-described risk factor for pyelonephritis. In some cases, repeated infection and/or transmission of high bladder pressures into the upper tracts in cases of secondary VUR can result in significant renal scarring and even progression to renal insufficiency [5•]. The prevention of the sequelae of recurrent pyelonephritis and renal scarring is the primary goal in the management of a patient with VUR and the impetus for treatment.

VUR is radiographically diagnosed on voiding cystourethrogram and is assigned a grade of severity ranging from I to $\mathrm{V}$ based on criteria defined by the International Reflux Committee (see Fig. 1) [6]. As suggested by this grading system, VUR exists on a spectrum. Higher grade, "clinically significant" VUR (grades III-V) is less likely to resolve on its own and, when it does, is estimated to take a longer period of time. Conversely, the natural history of low-grade VUR (grades I-II) is more rapid resolution in a higher percentage of patients. Resolution rates for grades I, II, III, and IV have been reported to be $83,77,68$, and $36 \%$ over an estimated median years to a resolution of $2.7,3.1,4.5$, and 9.5 years, respectively [7].

As attempts have been made to shift from diagnosing and treating all grades of VUR to a more selective strategy, multiple screening and diagnostic guidelines have been developed that are varied and conflicting [5•]. A spectrum of management options has also evolved, targeting UTI prevention, correction of VUR, or both. These include management of bowel and bladder dysfunction with various behavioral and pharmacologic interventions, antimicrobial prophylaxis, and finally surgical intervention with endoscopic injection, open, and robotic-assisted laparoscopic ureteral reimplantation.

In this review, we will summarize the operative and non-operative options for the management of primary VUR, highlighting our own philosophy and experiences as well as recent developments in literature. We hope that our discussion of VUR treatment options will provide insight for pediatricians practicing in the community as to why a certain management strategy might be selected for particular patient based on their individual circumstances.

\section{Treatment}

\section{Management of bowel and bladder dysfunction (BBD)}

- Surveillance alone is suboptimal in a child with VUR; all children diagnosed with VUR should be screened for bowel and bladder dysfunction (BBD) and toileting behaviors optimized. 


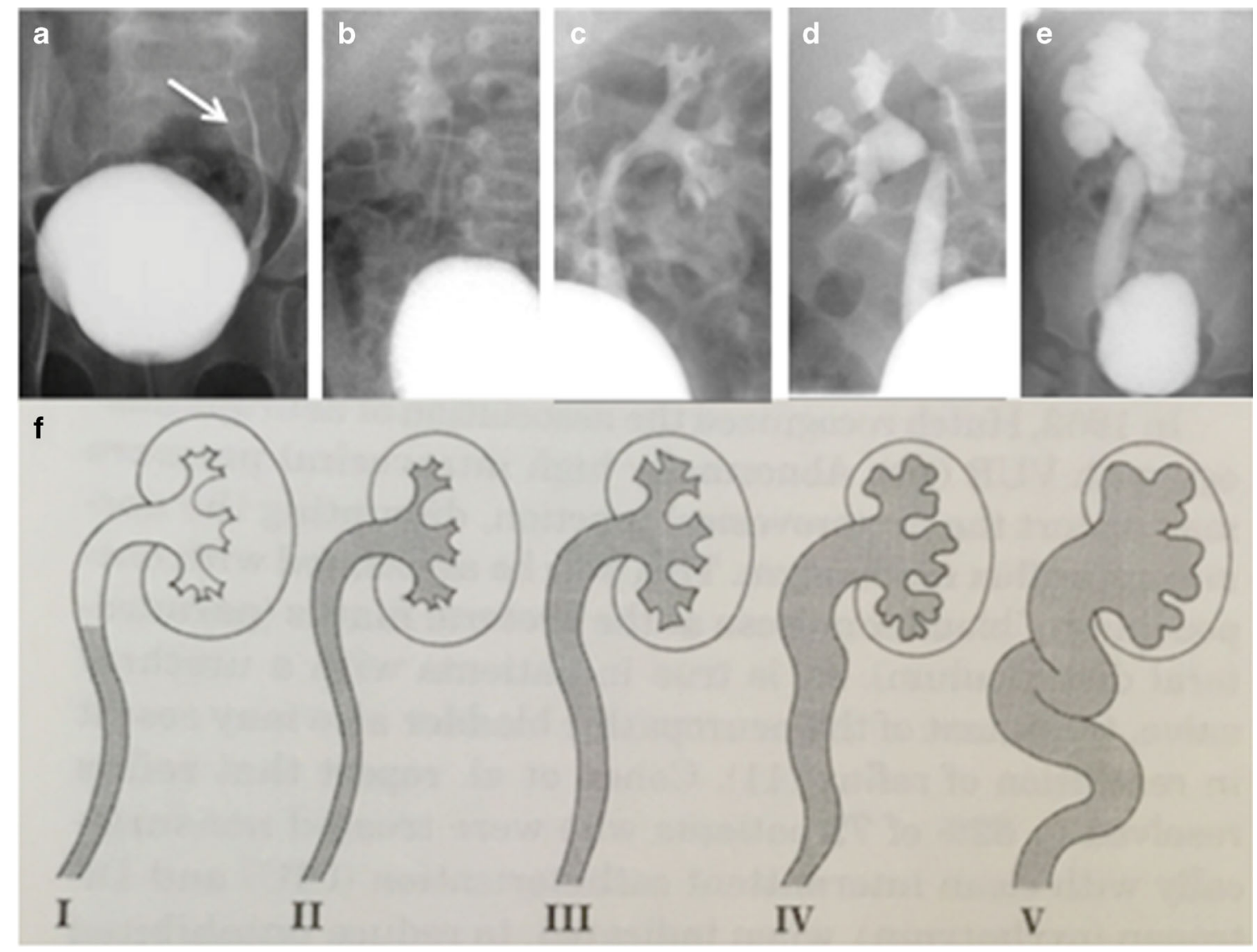

Fig. 1. Depiction of grade I-V vesicoureteral reflux (international reflux classification) on voiding cystourethrogram (a-e) and in diagram forms (f). a Left grade I (contrast in the ureter only). b Right grade II (contrast in the ureter and renal pelvis without calyceal dilation and normal calyceal fornices). $\mathrm{c}$ Left grade III (mild or moderate dilation of the ureter and renal pelvis. No or slight blunting of calyceal fornices). $\mathbf{d}$ Right grade IV (moderate dilation and/or tortuosity of the ureter, moderate renal pelvis, and calyceal dilation. Loss of the sharp angle of the fornices). e Right grade V (severe dilation and tortuosity of the ureter with severe dilation of the renal pelvis and calyces). $\mathbf{f}$ International reflux classification (modified with permission from Pediatric Nephrology, 4th Edition, 1999 Lippincott Williams \& Wilkins, Media, Pennsylvania).

- No single treatment regimen for BBD is recommended. Management may include dietary and behavioral modification, the use of anticholinergics, alpha-blockers, treatment of constipation, and in some cases pelvic floor therapy or biofeedback.

- Successful management of BBD decreases the risk of febrile UTI, facilitates the resolution of VUR, and can improve the rate of cure after endoscopic therapy.

BBD and VUR are closely intertwined. It is no coincidence that VUR resolves as children age, develop lower voiding pressures, and increase their bladder capacity [8]. Similarly, an increased risk for UTI is also well known to exist in children with coexisting BBD $[9,10]$. Although the natural history of VUR suggests a high likelihood of spontaneous resolution with maturation of bladder function, it is our opinion that surveillance alone is suboptimal. At a minimum, children presenting with VUR 
Antimicrobial prophylaxis

- Antimicrobial prophylaxis can minimize or eliminate febrile urinary tract infections in some children with VUR, and the benefit is greatest in older girls with higher grade VUR and concomitant BBD.

- The effect of prophylaxis on long-term renal scarring continues to be debated.

Antimicrobial prophylaxis (see Table 1) has historically been a standard therapy in prevention of UTI in children with any grade of VUR, based on the logic that the prevention of UTI would in turn prevent renal scarring until a child's reflux resolves or is surgically corrected. In an era of increasing antibiotic resistance, this assumption began to be questioned, particularly when compliance rates with prophylaxis were demonstrated to be as low as $40 \%$ [15]. An increasing number of studies challenged the effectiveness of antimicrobial prophylaxis, as summarized by a recent Cochrane review of 20 randomized control trials including 2324 children which concluded that long-term, low-dose antibiotic prophylaxis failed to significantly reduce symptomatic or febrile UTIs compared to placebo or no treatment [16]. 


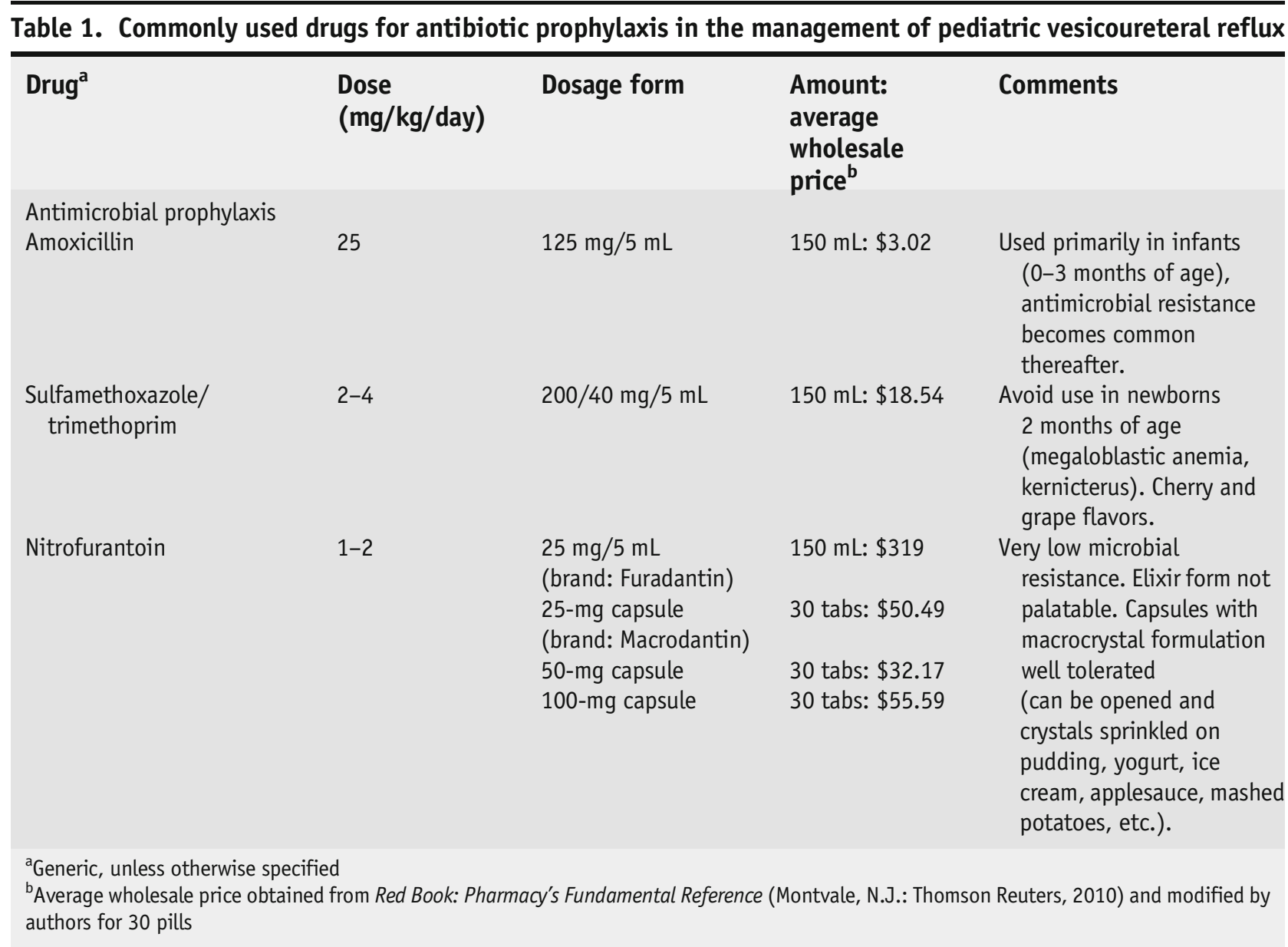

While an in-depth analysis of the vast body of literature on antimicrobial prophylaxis and VUR is beyond the scope of this review, it is unlikely that the effectiveness of antimicrobial prophylaxis in children with VUR is an all or nothing phenomenon. There are certain patients that do derive real benefit from antimicrobial prophylaxis as illustrated by the recently published Swedish reflux trial [17•] and RIVUR trial [18•].

The Swedish reflux trial randomized 203 children with grade 3-4 VUR and a history of "one to several" UTIs to antibiotic prophylaxis, endoscopic injection, or surveillance. At study entry, abnormal DMSA imaging suggestive of renal scarring was present in $61 \%$ of the children. Over the 2 year study period, UTI recurrence was lowest in girls on antibiotic prophylaxis (19\%) versus surveillance (57\%), and the occurrence of new renal scarring was significantly lower in girls on antibiotic prophylaxis. Not surprisingly, lower urinary tract dysfunction at follow-up was associated with a lower rate of improvement of VUR as well as an increased risk of recurrent infection. No difference was seen in boys, which the authors attribute to the overall decreased risk of UTI in boys after 6 months of age, whereas the risk of UTI in girls increases after 6 months. 
Alternatively, let's consider the recently completed randomized, double-blinded, placebo-controlled multicenter RIVUR trial. When comparing this patient population to that of the Swedish reflux trial, over half of the patients (54\%) had low grade 1-2 VUR, and almost all the patients in the study were enrolled after their first UTI ( $91.3 \%)$. Ninety-six percent of the patients in the RIVUR had no evidence of renal scarring at study intake. In this study, antibiotic prophylaxis reduced the risk of UTI recurrence by $50 \%$, and this benefit was greatest in children with concomitant BBD, where an $80 \%$ reduction in UTI recurrence was observed. Antibiotic prophylaxis did not, however, impact the formation of new renal scarring (10-12 \% occurrence) in either the prophylaxis or placebo groups, probably reflecting the fact that the majority of children had lower grade VUR and a history of only one febrile UTI. Also, the impact of rapid identification and treatment of recurrent febrile UTIs as a consequence of being a study participant may have been another protective factor.

In summary, antimicrobial prophylaxis most likely does not substantially benefit all patients with VUR, particularly those with low-grade VUR who have had only one UTI, and the individual characteristics of each patient must be considered in making the decision to place a child with VUR on prophylaxis. There clearly remains a subset of higher risk patients-recurrent UTIs, older girls with BBD, high-grade ( $\geq$ III) VUR, and/or evidence of renal scarring - in whom antibiotic prophylaxis can have a significant, beneficial impact. Furthermore, the clinician and parents must also weigh subjective factors including the severity and impact of recurrent UTIs on the family and the child and the parents' ability to comply with and the concerns about long-term use of antibiotics in this decision-making process.

- Circumcision can decrease the risk of UTIs in males with VUR, especially in infants less than 6 months of age.

- Endoscopic injection of dextranomer hyaluronic acid copolymer (Deflux) is a less invasive surgical alternative with the highest likelihood of success in children with low-grade (I-III) reflux with normal bladder and bowel function. Parents should be counseled on the potential need for additional investigation and intervention due to the lower success rates with this modality when compared with surgical ureteral reimplantation.

- Open ureteral reimplantation is the gold standard for the correction of VUR with success rates greater than $95 \%$.

- Robotic-assisted ureteral reimplantation is an increasingly utilized modality with success rates approaching open ureteral reimplantation in small patient series. Further studies are needed to define the costs and benefits of this procedure.

\section{Circumcision}

In febrile infants less than 6 months of age, uncircumcised males have been found to have a higher rate of UTI (21.3\%) compared to circumcised males 
$(2.3 \%)$ and females (5\%), translating to an approximately tenfold increase in risk for UTI [19]. Therefore, circumcision provides a substantial opportunity to mitigate the risk of further UTIs in an uncircumcised male infant with a history of UTI, particularly if that child has an additional risk factor for UTI such as VUR. The incidence of adverse events associated with newborn male circumcisions in US medical settings is low, with recent estimates of $0.5 \%$ or less [20]. The window of impact for circumcision, however, does appear to be finite. Studies have shown that if successful surgical correction of VUR is performed in an uncircumcised child, concomitant circumcision at the time of reflux surgery does not appear to impact the occurrence of febrile UTI [21]. The decision to pursue circumcision for one's child is a personal one and often multi-factorial; however, it is an important option to include in the counseling of the parents of an uncircumcised male infant with urinary tract infections and concomitant VUR. It should be noted that in many cases of boys with VUR, circumcision can replace the need for long-term antibiotic prophylaxis.

\section{Endoscopic injection}

In 2001, the US Food and Drug Administration approved the use of Deflux for endoscopic injection for the treatment of pediatric patients with primary grade 2-4 VUR. While multiple Deflux administration techniques exist, the most commonly one utilized by pediatric urologists today is the so-called double HIT (hydrodistention implantation technique) [22]. This technique utilizes both the proximal and distal intraureteral implantation sites with the goal of complete coaptation of both the ureteral orifice and intravesical ureteral tunnel [23].

Reported success rates of Deflux vary widely, and the factor most predictive of success is the pre-operative grade of VUR. In a recent systematic review, children with grade 1 VUR had an $81 \%$ probability of successful correction of VUR, while children with grade 5 VUR had only a $62 \%$ probability of success [24]. These findings are similar to an earlier meta-analysis which reported Deflux success rates of $78.5,78.5,72.31,62.5$, and $50.9 \%$ in children with grade I-V VUR, respectively [25].

Factors associated with decreased success of Deflux injection include concomitant anatomic and functional abnormalities of the bladder or previous surgical ureteral reimplantation [24]. Concerns also exist regarding the sustained success of Deflux with longer-term follow-up. At 1-year status postDeflux injection, Lee et al. reported only a $46 \%$ continued success rate [26]. The occurrence of recurrent febrile UTIs in patients after Deflux injection also appears to herald late failure; Sedberry-Ross et al. found that as many as $30 \%$ of high-risk patients may experience a febrile UTI after Deflux even when an initial post-procedure VCUG demonstrated successful treatment [27]. Of those patients, up to $92 \%$ demonstrated a recurrence of VUR when re-imaged. Seventyfive percent of those patients with a post-Deflux febrile UTI had concomitant dysfunctional elimination. Renal scarring and a prior history of multiple UTIs were also risk factors. Similarly, Chi et al. also found that $50 \%$ of patients presenting with a post-Deflux febrile UTI (10.5\%) had recurrent VUR [28].

While local, self-limiting symptoms may occur post-endoscopic injection, Deflux is a low-risk procedure. The most commonly occurring serious side effect is ureteral obstruction which has been documented to occur $0.6 \%$ of injected ureters. This obstruction is usually transient and can be successfully managed with temporary ureteral stenting. Also of note, nearly all patients experiencing 
this complication ( $80 \%$ ) have had dysfunctional voiding or a neuropathic bladder [29].

Due to the significant expense of the material, concern has been raised regarding the cost-effectiveness of Deflux injection therapy. A recent estimate noted the cost of a $1-\mathrm{mL}$ vial of Deflux to be $\$ 1045$. Furthermore, there has been a $33 \%$ increase in the mean number of vials utilized per procedure at multiple institutions over a 6-year period [30]. While it has been speculated that an increased amount of material injected might increase the effectiveness of the procedure or reflect changes in practice patterns such as increased treatment of bilateral or higher grades of VUR with Deflux, the exact reasons are unknown, and this remains an important consideration.

While Deflux is costly and has a lower rate of success when compared to surgical ureteral reimplantation, it remains a reasonable alternative particularly suited for children with mild to moderate low-grade VUR without concomitant BBD or anatomic bladder anomalies whose parents desire to avoid more invasive forms of surgical correction. We recommend follow-up with an ultrasound at 1-month post-endoscopic injection to rule out obstruction followed by a voiding cystourethrogram at 3 months postoperatively to ensure resolution of VUR. In patients with correction of their VUR at 3 months, an additional VCUG at 1 year postoperatively is ideal to monitor for late recurrences, but rarely is this done routinely unless febrile infection recurs. Many centers are now foregoing the post-Deflux VCUG and are only imaging those patients that present with a febrile UTI post-Deflux injection.

\section{Ureteral reimplantation}

While endoscopic injection corrects VUR by coapting the intravesical tunnel and decreasing the diameter of the intravesical ureter, surgical ureteral reimplantation corrects VUR by increasing the length of the intravesical tunnel. This can be achieved by both open and minimally invasive surgical techniques utilizing both intra- and extravesical approaches.

Absolute indications for surgical ureteral reimplantation include one or more breakthrough urinary tract infections while on prophylactic antibiotics associated with new renal damage on DMSA scan, both in cases where there is an inability to comply with therapy or when infections are caused by resistant organisms. However, one prospective study has shown that the actual risk of new renal damage on DMSA scans associated with a breakthrough febrile UTI is only $17 \%$ [31]. Therefore, in the absence of new renal damage, a change in antibiotic prophylaxis is a reasonable alternative, particularly if the patient has had only one breakthrough UTI. A relative indication for surgical reimplantation includes persistent moderate or higher grade VUR in an older child after an extended trial of antibiotic prophylaxis. In this scenario, concerns may arise related to long-term antibiotic exposure and accumulating radiation exposure from repeated surveillance imaging. The process of surveillance may in and of itself become tedious, leading parents to want to have the reflux "fixed."

\section{Open surgical techniques}

Open ureteral reimplantation is described as the gold standard for the surgical correction of VUR with success rates of greater than $95 \%$ in over 8000 ureters [32]. Immediate postoperative side effects of intravesical ureteral reimplants are 
well described-hematuria, bladder spasms, and irritative voiding symptoms-and are related to the need for an open cystotomy to perform reimplantation. These issues are usually transient but can be largely obviated by an extravesical approach to the surgical reimplant. Although extravesical ureteral reimplantation, particularly in the setting of bilateral reimplantation, has been associated anecdotally with urinary retention secondary to disruption of the nerve supply to the urinary bladder, use of a modified surgical technique limiting ureteral and detrusor dissection as well as a strict postoperative pathway involving early ambulation and patient education have markedly reduced its occurrence [33]. The complications of ureteral obstruction and recurrent VUR are rare $(<5 \%)$ with open reimplantation techniques. Open ureteral reimplantation is our preferred technique for any VUR that is not straightforward, for example, VUR associated with anatomic anomalies (duplication anomalies, diverticula) and in patients with a history of lower urinary tract dysfunction, either neuropathic or related to BBD.

\section{Robotic-assisted ureteral reimplantation}

Based on a review of the Pediatric Health Information System, a database containing information from 47 of the larger tertiary pediatric centers in the USA, the number of robotic pediatric urologic procedures performed is increasing at a rate of $17 \%$ per year [34]. Ureteral reimplants are second only to robotic-assisted laparoscopic pyeloplasty in this list.

Since first described by Peters in 2005, multiple groups have reported on their success with laparoscopic robotic-assisted extra- and intra-vesical ureteral reimplantation, with success rates ranging from 83 to $95 \%$ in relatively small series of patients $[35,36]$. In contrast, a recent multi-institutional review of 61 patients (93 ureters) treated with extravesical robotic-assisted ureteral reimplantations performed by five robotically experienced pediatric urologists found a $23 \%$ failure rate, with $10 \%$ of the patients experiencing surgical complications or requiring an additional procedure for persistent VUR [37].

The cost-effectiveness of pediatric urologic robotic surgery continues to be explored. Although hospital stays are shorter in robotic-assisted ureteral reimplants when compared to their open counterparts, the cost of these hospitalizations at present appears to be higher. Other potential benefits to roboticassisted surgery include decreased postoperative pain. One small comparative study of postoperative pain in children undergoing open versus robotic-assisted ureteral reimplantation found decreased narcotic analgesic requirements in children undergoing robotic surgery, and, while a difference in pain scores was not significantly different between the groups, the percentage of children experiencing severe pain was less in the robotic-assisted group (9\% versus $45 \%$ ) [38]. This has been confirmed by other series [39]. In addition to limiting postoperative pain, particularly in older patients, the smaller incisions from robotic-assisted ureteral reimplantation may also provide an improved cosmetic outcome from the perspective of patients and their parents. After showing parents and children older than 7 years of age images of scars for robotic versus open ureteral reimplantation, $85 \%$ preferred robotic scars, provided that the surgical procedure was equally efficacious [40].

While robotic ureteral reimplantation demonstrates promise and some series have approached the success rates of open ureteral reimplantation, patient numbers are small and initial results need confirmation by larger 
comparative series. The standardization of the technique also remains unclear in the face of varying inter-institutional outcomes.

\section{Conclusions/emerging therapies}

While no new techniques in the management of VUR have emerged since the advent of robotic-assisted ureteral reimplantation in the mid-2000s, the "best" management strategy for pediatric VUR remains a topic of discussion. Ongoing issues include the role of antimicrobial prophylaxis in specific VUR patient subpopulations, the ideal indications for Deflux treatment, and the costeffectiveness of robotic-assisted techniques, particularly in light of recent data regarding the relative success rate compared to gold standard, open ureteral reimplantation.

\section{Compliance with ethical standards}

\section{Conflict of interest}

Tanya D. Davis declares that she has no conflict of interest.

H. Gil Rushton declares that he has no conflict of interest.

Human and animal rights and informed consent

This article does not contain any studies with human or animal subjects performed by any of the authors.

\section{References and Recommended Reading}

Papers of particular interest, published recently have been highlighted as:

- Of importance

$\bullet \quad$ Of major importance

1. Sargent MA. What is the normal prevalence of vesicoureteral reflux? Pediatr Radiol. 2000;30:587-93.

2. Park JM. Vesicoureteral reflux: anatomic and functional basis of etiology. In: Docimo SG, editor. The KelaisKing-Belman Textbook of Clinical Pediatric Urology. Fifthth ed. United Kingdom: Informa UK Ltd; 2007. p. 655-62.

3. $\quad$ Nguyen HT, Herndon CD, Cooper C, Gatti J, Kirsch A, Kokorowski $\mathrm{P}$, et al. The society for fetal urology consensus statement on the evaluation and management of antenatal hydronephrosis. J Pediatr Urol. 2010;6:212-31.

An in-depth summary of the evaluation and management of the common causes of antenatal hydronephrosis, including but not limited to vesicoureteral reflux.

4. Hollowell JG, Greenfield SP. Screening siblings for vesicoureteral reflux. J Urol. 2002;168:2138-41.

5. $\quad$ Routh JC, Bogaert GA, Kaefer M, Manzoni G, Park JM, Retik $\mathrm{AB}$, et al. Vesicoureteral reflux: current trends in diagnosis, screening, and treatment. Eur Urol. 2012;61:773-82.
Provides a concise summary of current trends and controversies in the diagnosis of vesicoureteral reflux.

6. Lebowitz RL, Olbing H, Parkkulainen KV, Smellie JM, Tamminen- Möbius TE. International system of radiographic grading of vesicoureteric reflux. International Reflux Study in Children. Pediatr Radiol. 1985;15:105-9.

7. Schwab Jr CW, Wu HY, Selman H, Smith GH, Snyder 3rd HM, Canning DA. Spontaneous resolution of vesicoureteral reflux: a 15-year perspective. J Urol. 2002;168:2594-9.

8. Chandra M, Maddix H, McVicar M. Transient urodynamic dysfunction of infancy: relationship to urinary tract infections and vesicoureteral reflux. J Urol. 1996;155:673-7.

9. Van Batavia JP, Ahn JJ, Fast AM, Combs AJ, Glassberg KI. Prevalence of urinary tract infection and vesicoureteral reflux in children with lower urinary tract dysfunction. J Urol. 2013;190(4 Suppl):1495-9.

10. Chen JJ, Wenyang M, Homayoon K. A multivariate analysis of dysfunctional elimination syndrome and its 
relationships with gender, urinary tract infection and vesicoureteral reflux in children. J Urol. 2004;171:1907.

11. Elder JS, Diaz M. Vesicoureteral reflux-the role of bladder and bowel dysfunction. Nat Rev Urol. 2013;10:640-8.

A concise summary on the interplay between VUR, BBD, and UTIs, as well as the evaluation of BBD in the pediatric patient.

12.• Peters CA, Skoog SJ, Arant Jr BS, Copp HL, Elder JS, Guy Hudson R, et al. Summary of the AUA guideline on the management of primary vesicoureteral reflux in children. J Urol. 2010;184:1134-44.

A summary of the American Urologic Associations recommendations to urologists on the management of primary vesicoureteral reflux in children. The most comprehensive review available to date on the management of primary vesicoureteral reflux.

13. De Paepe H, Renson C, Van Laecke E, Raes A, Vande Walle J, Hoebeke P. Pelvic-floor therapy and toilet training in young children with dysfunctional voiding and obstipation. BJU Int. 2000;85:889-93.

14. Loening-Baucke V. Urinary incontinence and urinary tract infection and their resolution with treatment of chronic constipation of childhood. Pediatrics. 1997; 100:228-32.

15. Copp HL, Nelson CP, Shorliffe LD, Lai J, Saigal CS, Kennedy WA. Compliance with antibiotic prophylaxis in children with vesicoureteral reflux: results from a national pharmacy claims database. J Urol. 2010;183:1994-9.

16. Nagler EV, Williams G, Hodson EM, Craig JC. Interventions for primary vesicoureteric reflux. Cochrane Database Syst Rev. 2011;6, CD001532.

17. Brandstrom P, Jodal U, Sillen U, Hansson S. The Swedish reflux trial: review of a randomized, controlled trial in children with dilating vesicoureteral reflux. J Ped Urol. 2011;7:594-600.

A study of antimicrobial prophylaxis that illustrates the importance of this therapy in certain high-risk groups, namely girls with evidence of renal scarring who have recurrent urinary tract infections and higher grade reflux in the setting of concomitant bowel and bladder dysfunction.

18. The RIVUR Trial Investigators. Antimicrobial prophylaxis for children with vesicoureteral reflux. $\mathrm{N}$ Engl J Med. 2014;370:2367-76.

Recent well-designed multicenter randomized control trial that also highlights the important role that antimicrobial prophylaxis can play in certain subgroups of patients with VUR.

19. Zorc JJ, Levine DA, Platt SL, Dayan PS, Macias CG, Krief $\mathrm{W}$, et al. Multicenter RSV-SBI study group of the pediatric emergency medicine collaborative research committee of the American Academy of Pediatrics. Clinical and demographic factors associated with urinary tract infection in young febrile infants. Pediatrics. 2005; 116:644-8.

20. El Bcheraoui C, Zhang X, Cooper CS, Rose CE, Kilmarx $\mathrm{PH}$, Chen RT. Rates of adverse events associated with male circumcision in U.S. medical settings, 2001 to 2010. JAMA Pediatr. 2014;168:625-34.
21. Kwak C, Oh SJ, Lee A, Choi H. Effect of circumcision on urinary tract infection after successful antireflux surgery. BJU Int. 2004;94(4):627-9. Epub 2004/08/27.

22. Kirsch AJ, Arlen AM, Lackgren G. Current trends in dextranomer hyaluronic acid copolymer (Deflux) injection technique for endoscopic treatment of vesicoureteral reflux. Urology. 2014;84:462-8.

23. Cerwinka W, Scherz HC, Kirsch AJ. Dynamic hydrodistention classification of the ureter and the double HIT method to correct vesicoureteral reflux. Arch Esp Urol. 2008;61:882-7.

24. Routh JC, Inman BA, Reinberg Y. Detranomer/ hyaluronic acid for pediatric vesicoureteral reflux: systematic review. Pediatrics. 2010;5:1010-9.

25. Elder JS, Diaz M, Caldamone AA, Cendron M, Greenfield S, Hurwitz F, et al. Endoscopic therapy for vesicoureteral reflux: a meta-analysis. Reflux resolution and urinary tract infection. J Urol. 2006;175:716.

26. Lee EK, Gatti JM, Demarco RT, Murphy JP. Long-term followup of dextranomer/hyaluronic acid injection for vesicoureteral reflux: late failure warrants continued followup. J Urol. 2009;181(4):1869-74.

27. Sedberry-Ross S, Rice DC, Pohl HG, Belman AB, Majd $M$, Rushton HG. Febrile urinary tract infections in children with an early negative voiding cystourethrogram after treatment of vesicoureteral reflux with dextranomer/hyaluronic acid. J Urol. 2008;180:1605-10.

28. Chi A, Gupta A, Snodgrass W. Urinary tract infection following successful dextranomer/hyaluronic acid injection for vesicoureteral reflux. J Urol. 2008;179:1966.

29. Vandersteen DR, Routh JC, Kirsch AJ, Scherz HC, Ritchey ML, Shapiro E, et al. Post-operative ureteral obstruction after subureteric injection of dextranomer/hyaluronic acid copolymer. J Urol. 2006;176:1593-5.

30. Sorensen MD, Koyle MA, Cowan CA, Zamilpa I, Shnorhavorian M, Lendvay TS. Injection volumes of dextranomer/hyaluronic acid are increasing in the endoscopic management of vesicoureteral reflux. Pediatr Surg Int. 2010;26(5):509-13.

31. Szlyk GR, Williams SB, Majd M, Belman AB, Rushton HG. Incidence of new renal parenchymal inflammatory changes following breakthrough urinary tract infections in patients with vesicoureteral reflux treated with antibiotic prophylaxis: evaluation by $99 \mathrm{~m}$-technetium dimercapto-succinic acid renal scan. J Urol. 2003;170:1566-9.

32. Elder JS, Peters CA, Arant Jr BS, et al. Pediatric vesicoureteral reflux guidelines panel summary report on the management of primary vesicoureteral reflux in children. J Urol. 1997;157:1856-51.

33. Palmer JS. Bilateral extravesical ureteral reimplantation in toilet-trained children: short-stay procedure without urinary retention. Urology. 2009;73(2):285-8.

34. Mahida JB, Cooper JN, Herz D, Diefenbach KA, Deans $\mathrm{KJ}$, Minneci PC, et al. Utilization and costs associated 
with robotic surgery in children. J Surg Res. 2015. doi:10.1016/j.jss.2015.04.087.

35. Peters CA, Woo R. Intravesical robotically assisted bilateral ureteral reimplantation. J Endourol. 2005;19:618.

36. Weiss DA, Shukla AR. The robotic-assisted ureteral reimplantation: the evolution to a new standard. 2015. Urol Clin N Am. 2015;42:99-109.

37. Grimsby GW, Dwyer ME, Jacobs MA, Ost MC, Schneck FX, Cannon GM, et al. Multi-institutional review of outcomes of robotic-assisted laparoscopic extravesical ureteral reimplantation. J Urol. 2015;193:1791-5.
38. Harel M, Herbst KW, Silvis R, Makari JH, Ferrer FA, Kim C. Objective pain assessment after ureteral reimplantation: comparison of open versus robotic approach. J Pediatr Urol. 2015;11(2):82.e1-8.

39. Schomburg JL, Haberman K, Willihnganz-Lawson $\mathrm{KH}$, Shukla AR. Robotic-assisted laparoscopic ureteral reimplantation: a single surgeon comparison to open surgery. J Ped Urol. 2014;10:875-9.

40. Barbosa JA, Barayan G, Gridley CM, Sanchez DC, Passerotti CC, Houck CS, et al. Parent and patient perceptions of robotic vs open urological surgery scars in children. J Urol. 2013;190(1):244-50. 